PPPL-3448

PPPL-3448

UC-70

Macroscopic Description of Pressure-anisotropy-driven Collective Instability in Intense Charged Particle Beams

by

Sean Strasburg and Ronald C. Davidson

June 2000
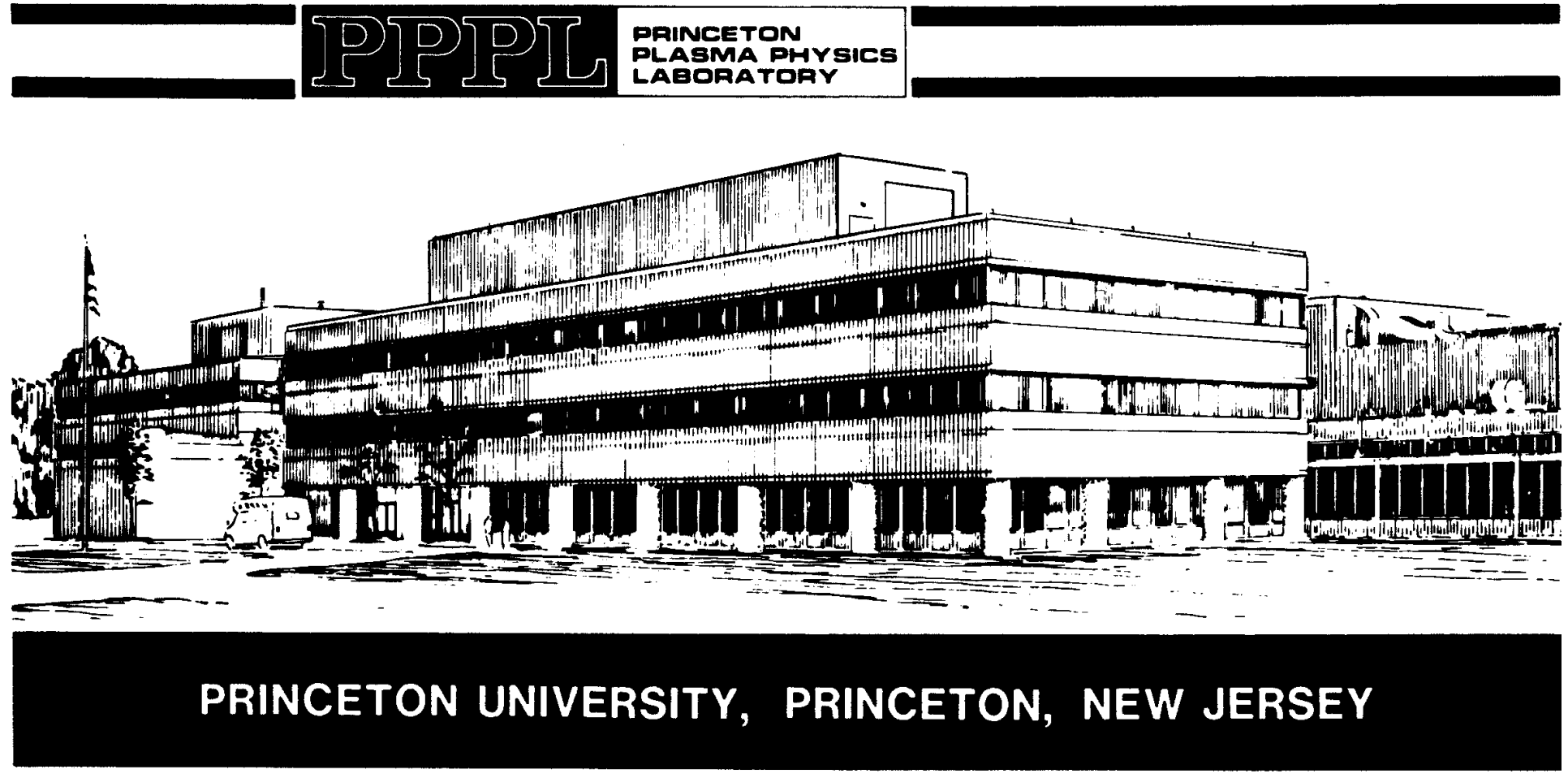


\section{PPPL Reports Disclaimer}

This report was prepared as an account of work sponsored by an agency of the United States Government. Neither the United States Government nor any agency thereof, nor any of their employees, makes any warranty, express or implied, or assumes any legal liability or responsibility for the accuracy, completeness, or usefulness of any information, apparatus, product, or process disclosed, or represents that its use would not infringe privately owned rights. Reference herein to any specific commercial product, process, or service by trade name, trademark, manufacturer, or otherwise, does not necessarily constitute or imply its endorsement, recommendation, or favoring by the United States Government or any agency thereof. The views and opinions of authors expressed herein do not necessarily state or reflect those of the United States Government or any agency thereof.

\section{Availability}

This report is posted on the U.S. Department of Energy's Princeton Plasma Physics Laboratory Publications and Reports web site in Calendar Year 2000. The home page for PPPL Reports and Publications is: http://www.pppl.gov/pub_report/

DOE and DOE Contractors can obtain copies of this report from:

U.S. Department of Energy

Office of Scientific and Technical Information

DOE Technical Information Services (DTIS)

P.O. Box 62

Oak Ridge, TN 37831

Telephone: (865) 576-8401

Fax: (865) 576-5728

Email: reports@adonis.osti.gov

This report is available to the general public from:

National Technical Information Service

U.S. Department of Commerce

5285 Port Royal Road

Springfield, VA 22161

Telephone: $1-800-553-6847$ or

(703) $605-6000$

Fax: (703) 321-8547

Internet: http://www.ntis.gov/ordering.htm 


\title{
Macroscopic Description of Pressure-Anisotropy-Driven Collective Instability in Intense Charged Particle Beams
}

\author{
Sean Strasburg and Ronald C. Davidson \\ Plasma Physics Laboratory \\ Princeton University \\ Princeton, New Jersey, 08543
}

\begin{abstract}
The macroscopic warm-fluid model developed by Lund and Davidson [Phys. Plasmas 5, 3028 (1998)] is used in the smooth-focusing approximation to investigate detailed stability properties of an intense charged particle beam with pressure anisotropy, assuming small-amplitude electrostatic perturbations about a waterbag equilibrium. Stability properties are calculated numerically for the case of extreme pressure anisotropy with $P_{\|}^{0}(r)=0$ and $P_{\perp}^{0}(r) \neq 0$, assuming axisymmetric wave perturbations $(\partial / \partial \theta=0)$ of the form $\delta \phi(\mathbf{x}, t)=\delta \hat{\phi}(r) \exp \left(i k_{z} z-i \omega t\right)$, where $k_{z}$ is the axial wavenumber, and $\operatorname{Im} \omega>0$ corresponds to instability (temporal growth). For sufficiently large values of $k_{z} r_{b}$, where $r_{b}$ is the beam radius, the analysis leads to an anisotropy-driven instability $(\operatorname{Im} \omega>0)$ provided the normalized Debye length $\left(\Gamma_{D}=\lambda_{D \perp} / r_{b}\right)$ is sufficiently large and the normalized beam intensity $\left(s_{b}=\hat{\omega}_{p b}^{2} / 2 \gamma_{b}^{2} \omega_{\beta \perp}^{2}\right)$ is sufficiently below the space-charge limit.
\end{abstract}




\section{INTRODUCTION}

There is increasing interest in the equilibrium and stability properties of intense charged particle beams ${ }^{1-3}$, with potential applications including heavy ion fusion, transmutation of radioactive waste, accelerator-based production of tritium, and spallation neutron sources. At the beam intensities of practical interest, it is particularly important to develop an improved theoretical understanding of the influence of space-charge effects and collective processes on detailed stability and transport properties ${ }^{4-20}$. A complete description of collective processes in intense nonneutral beams generally requires a knowledge of the beam distribution function $f(\mathbf{x}, \mathbf{p}, t)$ in the six-dimensional phase space $(\mathbf{x}, \mathbf{p})$. While considerable progress has been made in analytical investigations based on the Vlasov-Maxwell equations ${ }^{4-17}$, such kinetic analyses are often complex, even under idealized assumptions. It is therefore important to develop and test the robustness of alternative theoretical models, such as macroscopic models ${ }^{21-26}$ based on the fluid-Maxwell equations, for investigating beam equilibrium and stability properties.

Macroscopic fluid descriptions ${ }^{21-26}$ have met with recent success in describing the propagation of space-charge-dominated (low-emittance) beams in periodic-focusing transport systems ${ }^{22,25}$, and in describing high-frequency collective oscillations in high-intensity beams ${ }^{24}$. In this Letter, we make use of the macroscopic warm-fluid model developed by Lund and Davidson ${ }^{24}$ in the smooth-focusing approximation to investigate the linear stability properties of an intense charged particle beam, allowing for equilibrium pressure anisotropy $\left(P_{\perp}^{0} \neq P_{\|}^{0}\right)$. A particular focus in the present analysis is application of the warm-fluid model to investigate the anisotropy-driven $\left(P_{\perp}^{0}>P_{\|}^{0}\right)$ instability observed by Lund, et al. ${ }^{18^{-20}}$ in particle-in-cell simulations and studied analytically using the Vlasov-Maxwell equations. Such anisotropies are well known to develop naturally in accelerators. For example, for a beam of charged particles of mass $m$ and charge $q$ that is accelerated through a voltage $V$, a simple estimate shows that the final and initial longitudinal temperatures (in energy units) are related ${ }^{4}$ in the nonrelativistic case by $T_{\| f}=T_{\| i}^{2} / 2 q V$. In the relativistic case, this 
relation is modified to become $T_{\| f}=T_{\| i}^{2} \gamma_{i}^{3} / \beta_{f}^{2} \gamma_{f}^{3} m c^{2}$, where $\gamma$ is the relativistic mass factor and $\beta$ is the relativistic velocity. As an example, for an electron beam with initial energy 10 $\mathrm{keV}$ and temperature $T_{\| i}=0.5 \mathrm{eV}$ accelerated to $1 \mathrm{MeV}$, the final longitudinal temperature is $T_{\| f}=2.1 \times 10^{-8} \mathrm{eV}$, a decrease by seven orders-of-magnitude. In addition, the beam's effective transverse temperature $T_{\perp}$ and emittance are subject to increase due to nonlinearities in applied and self-field forces, nonstationary beam profiles, and mismatches, which may produce negligible changes in the parallel temperature. This simultaneous cooling in the parallel direction and heating in the transverse direction can provide the free energy to drive collective instabilities and cause a further deterioration in beam quality through the instability mechanism described in this paper. As a general remark, application of a warmfluid model to describe the equilibrium and stability properties of intense charged particle beams appears to be a remarkably robust and simple approach, both for the case of stable high-frequency collective oscillations ${ }^{24}$, as well as the unstable case considered here, where the instability is driven by gross macroscopic properties of the beam equilibrium (pressure anisotropy).

To briefly summarize the assumptions and macroscopic warm-fluid model, the present analysis considers an intense nonneutral beam consisting of charged particles with charge $q$ and rest mass $m$ propagating in the $z$-direction with average axial velocity $V_{b}=$ const., and characteristic directed kinetic energy $\left(\gamma_{b}-1\right) m c^{2}$ in the laboratory frame. Here, $\gamma_{b}=$ $\left(1-V_{b}^{2} / c^{2}\right)^{-1 / 2}$ is the relativistic mass factor, $c$ is the speed of light in vacuo, and a perfectly conducting cylindrical wall is located at radius $r=r_{w}$, where $r=\left(x^{2}+y^{2}\right)^{1 / 2}$ is the radial distance from the beam axis. The characteristic beam radius is denoted by $r_{b}$, and it is assumed that the particle motion in the beam frame is nonrelativistic. Transverse confinement of the beam particles is provided by applied magnetic or electric focusing fields, and in the smooth-focusing approximation we model the applied transverse focusing force on a beam particle by $\mathbf{F}_{f o c}=-\gamma_{b} m \omega_{\beta \perp}^{2}\left(x \hat{\mathbf{e}}_{x}+y \hat{\mathbf{e}}_{y}\right)$, where $\omega_{\beta \perp}=$ const. is the effective betatron frequency for the transverse oscillations, and $(x, y)$ is the transverse displacement from the beam axis. 
The present analysis is carried out in the electrostatic approximation, where the selfelectric field $\mathbf{E}^{s}(\mathbf{x}, t)$ produced by the beam space charge is $\mathbf{E}^{s}=-\nabla \phi$, and the electrostatic potential $\phi(\mathrm{x}, t)$ is determined self-consistently from Poisson's equation, $\nabla^{2} \phi=-4 \pi q n(\mathrm{x}, t)$, where $n(\mathrm{x}, t)$ is the number density. In addition, the axial beam current, $q n(\mathrm{x}, t) V_{z}(\mathrm{x}, t)$, where $V_{z}(\mathrm{x}, t)$ is the average axial velocity, produces a transverse self-magnetic field, $\mathbf{B}^{s}(\mathrm{x}, t)$, where $\mathbf{B}^{s}=\nabla A_{z} \times \hat{\mathbf{e}}_{z}$. Here, $A_{z}(\mathrm{x}, t)$ is determined self-consistently in the magnetostatic approximation from Maxwell's equation, $\nabla^{2} A_{z}=-(4 \pi / c) q n V_{z}$. In circumstances where the average axial velocity is approximately uniform over the beam cross section with $V_{z} \simeq V_{b}=$ const., which we assume to be the case, the self-field potentials, $\phi(\mathrm{x}, t)$ and $A_{z}(\mathrm{x}, t)$, are related by the familiar expression, $A_{z}=\left(V_{b} / c\right) \phi$.

To describe the dynamics of the intense charged particle beam interacting with the applied focusing field and the self-generated electric and magnetic fields, we make use of the macroscopic warm-fluid model developed by Lund and Davidson ${ }^{24}$. To briefly summarize, by taking appropriate momentum moments of the nonlinear Vlasov equation for the beam distribution function $f(\mathrm{x}, \mathrm{p}, t)$, we obtain an interconnected chain of macroscopic fluid equations ${ }^{21}$ advancing the particle density $n(\mathrm{x}, t)$, the average flow velocity $\mathbf{V}(\mathbf{x}, t)=V_{z}(\mathrm{x}, t) \hat{\mathbf{e}}_{z}+\mathbf{V}_{\perp}(\mathrm{x}, t)$, the pressure tensor $\mathbf{P}(\mathrm{x}, t)$, the heat flow tensor $\mathbf{Q}(\mathrm{x}, t)$, etc. Following Lund and Davidson ${ }^{24}$, we adopt a model in which the heat-flow contribution, proportional to $(\partial / \partial \mathbf{x}) \cdot \mathbf{Q}(\mathbf{x}, t)$, is neglected in the dynamical equation advancing the pressure tensor $\mathbf{P}(\mathrm{x}, t)$. In addition, we adopt a model ${ }^{21}$ in which $\mathbf{P}(\mathbf{x}, t)$ is assumed to be isotropic in the plane perpendicular to the beam propagation direction (the $z$-direction), i.e., $\mathbf{P}(\mathbf{x}, t)=P_{\perp}(\mathbf{x}, t)\left(\hat{\mathbf{e}}_{x} \hat{\mathbf{e}}_{x}+\hat{\mathbf{e}}_{y} \hat{\mathbf{e}}_{y}\right)+P_{\|}(\mathbf{x}, t) \hat{\mathbf{e}}_{z} \hat{\mathbf{e}}_{z}$, where $P_{\perp}(\mathbf{x}, t)$ and $P_{\|}(\mathbf{x}, t)$ are scalar pressures. In this case, making use of the assumptions enumerated earlier, the warm-fluid equations $^{24}$ appropriately generalized to the case of relativistic axial motion are given by the continuity equation for $n(\mathrm{x}, t)$,

$$
\left(\frac{\partial}{\partial t}+V_{z} \frac{\partial}{\partial z}+\mathbf{V}_{\perp} \cdot \frac{\partial}{\partial \mathbf{x}_{\perp}}\right) n+n\left(\frac{\partial V_{z}}{\partial z}+\frac{\partial}{\partial \mathbf{x}_{\perp}} \cdot \mathbf{V}_{\perp}\right)=0
$$

the perpendicular force balance equation for $\mathbf{V}_{\perp}(\mathbf{x}, t)$, 


$$
\gamma_{b} m n\left(\frac{\partial}{\partial t}+V_{z} \frac{\partial}{\partial z}+\mathbf{V}_{\perp} \cdot \frac{\partial}{\partial \mathbf{x}_{\perp}}\right) \mathbf{V}_{\perp}+\frac{\partial}{\partial \mathbf{x}_{\perp}} P_{\perp}=-n q \frac{1}{\gamma_{b}^{2}} \nabla_{\perp} \phi-\gamma_{b} m n \omega_{\beta \perp}^{2} \mathbf{x}_{\perp}
$$

the parallel force balance equation for $V_{z}(\mathrm{x}, t)$,

$$
\gamma_{b} m n\left(\frac{\partial}{\partial t}+V_{z} \frac{\partial}{\partial z}+\mathbf{V}_{\perp} \cdot \frac{\partial}{\partial \mathbf{x}_{\perp}}\right) V_{z}+\frac{\partial}{\partial z} P_{\|}=-n q \frac{\partial \phi}{\partial z}
$$

the equation of state for the perpendicular pressure $P_{\perp}(\mathrm{x}, t)$,

$$
\left(\frac{\partial}{\partial t}+V_{z} \frac{\partial}{\partial z}+\mathbf{V}_{\perp} \cdot \frac{\partial}{\partial \mathbf{x}_{\perp}}\right)\left(\frac{P_{\perp}}{n^{2}}\right)-\frac{P_{\perp}}{n^{2}} \frac{\partial V_{z}}{\partial z}=0
$$

the equation of state for the parallel pressure $P_{\|}(\mathrm{x}, t)$,

$$
\left(\frac{\partial}{\partial t}+V_{z} \frac{\partial}{\partial z}+\mathbf{V}_{\perp} \cdot \frac{\partial}{\partial \mathbf{x}_{\perp}}\right)\left(\frac{P_{\|}}{n}\right)+\frac{2 P_{\|}}{n} \frac{\partial V_{z}}{\partial z}=0
$$

and Poisson's equation for the electrostatic potential $\phi(\mathrm{x}, t)$,

$$
\nabla_{\perp}^{2} \phi+\frac{\partial^{2}}{\partial z^{2}} \phi=-4 \pi q n
$$

Equations (1)-(6) provide a closed macroscopic description of the nonlinear evolution of the beam interacting with the applied focusing field and the self-generated electric and magnetic fields, $\mathbf{E}^{s}=-\nabla \phi$ and $\mathbf{B}^{s}=\left(V_{b} / c\right) \nabla \phi \times \hat{\mathbf{e}}_{z}$. In obtaining Eqs. (1)-(6), it has been assumed that the fluid motions in the beam frame are nonrelativistic, i.e., $\mathbf{V}_{\perp}^{2} / c^{2},\left(V_{z}-\right.$ $\left.V_{b}\right)^{2} / c^{2}, P_{\perp} / \gamma_{b} n m c^{2}, P_{\|} / \gamma_{b} n m c^{2} \ll 1$

Equations (1)-(6) can be used to investigate detailed macroscopic equilibrium and stability properties for perturbations about a wide range of beam equilibria ${ }^{24}$ ranging from a warmfluid thermal equilibrium with diffuse radial density profile, to a warm-fluid KapchinskijVladimirskij (KV) equilibrium with step-function density profile, to a warm-fluid waterbag equilibrium. For example, assuming $\partial / \partial z=0$, Lund and Davidson have investigated ${ }^{24}$ sta- $^{-}$ ble electrostatic oscillations for perturbations about a warm-fluid KV equilibrium. In the present analysis, allowing for a pressure anisotropy with $P_{\perp} \neq P_{\|}$and perturbations with $\partial / \partial z \neq 0$, we examine detailed stability properties for perturbations about a warm-fluid waterbag equilibrium ${ }^{11,13,24}$. 
Under steady-state (equilibrium) conditions with $\partial / \partial t=0$, we assume a matched, axisymmetric beam in which all equilibrium profiles (denoted with a superscript zero), satisfy $\partial / \partial \theta=0=\partial / \partial z=0$, and depend only on the radial distance $r=\left(x^{2}+y^{2}\right)^{1 / 2}$ from the beam axis. In equilibrium, it is further assumed that there is no perpendicular motion of the beam and that the axial flow velocity is uniform over the beam cross section, i.e., $\mathbf{V}_{\perp}^{0}=0$, and $V_{z}^{0}=V_{b}=$ const. For a warm-fluid waterbag equilibrium ${ }^{11,13,24}$, we assume that the perpendicular and parallel pressures are of the form

$$
P_{\perp}^{0}(r)=\left(\hat{T}_{\perp} / \hat{n}\right)\left[n^{0}(r)\right]^{2}, \quad P_{\|}^{0}(r)=\hat{T}_{\|} n^{0}(r)
$$

where $n^{0}(r)$ is the equilibrium density profile. In Eq. $(7), \hat{n}=n^{0}(r=0)=$ const. is the on-axis density, and the constants $\hat{T}_{\perp}$ and $\hat{T}_{\|}$are the perpendicular temperature and parallel temperature, respectively, at $r=0$, expressed in energy units. From Eq. (7), we note that the effective temperature profiles, $T_{\perp}^{0}(r)=P_{\perp}^{0}(r) / n^{0}(r)$ and $T_{\|}^{0}(r)=P_{\|}^{0}(r) / n^{0}(r)$, are given by $T_{\perp}^{0}(r)=\hat{T}_{\perp} n^{0}(r) / \hat{n}$ and $T_{\|}^{0}(r)=\hat{T}_{\|}=$const. That is, $T_{\perp}^{0}(r)$ has the same radial shape as the density profile $n^{0}(r)$, whereas $T_{\|}^{0}(r)$ is uniform (isothermal) over the beam cross section. We introduce the effective perpendicular Debye length $\lambda_{D \perp}$ and self-field intensity parameter $s_{b}$ defined by

$$
\lambda_{D \perp}^{2}=\frac{2 \hat{T}_{\perp} \gamma_{b}^{2}}{4 \pi q^{2} \hat{n}}, \quad s_{b}=\frac{\hat{\omega}_{p b}^{2}}{2 \gamma_{b}^{2} \omega_{\beta \perp}^{2}}
$$

where $\hat{\omega}_{p b}^{2}=4 \pi \hat{n} q^{2} / \gamma_{b} m$ is the on-axis plasma frequency-squared. Without presenting algebraic details, the exact solution for the equilibrium density profile is given by ${ }^{26}$

$$
n^{0}(r)= \begin{cases}\hat{n} \frac{I_{0}\left(r_{b} / \lambda_{D \perp}\right)-I_{0}\left(r / \lambda_{D \perp}\right)}{I_{0}\left(r_{b} / \lambda_{D \perp}\right)-1}, & 0 \leq r<r_{b}, \\ 0, & r_{b}<r \leq r_{w}\end{cases}
$$

where $r_{b}$ is the outer radius of the beam, and $r_{w}$ is the conducting wall radius. In terms of $\lambda_{D \perp}$ and $s_{b}=\hat{\omega}_{p b}^{2} / 2 \gamma_{b}^{2} \omega_{\beta \perp}^{2}$, the beam radius $r_{b}$ in Eq. (9) is determined self-consistently from

$$
I_{0}\left(r_{b} / \lambda_{D \perp}\right)=\frac{1}{1-\hat{\omega}_{p b}^{2} / 2 \gamma_{b}^{2} \omega_{\beta \perp}^{2}} .
$$


Here, $I_{0}(x)$ is the modified Bessel function of the first kind of order zero.

For the equilibrium density profile $n^{0}(r)$ specified by Eq. (9), we note that the density profile decreases monotonically from the on-axis value $n^{0}(r=0)=\hat{n}$ at $r=0$, to $n^{0}\left(r=r_{b}\right)=0$ at the beam edge $\left(r=r_{b}\right)$. At low beam intensities with $s_{b}=\hat{\omega}_{p b}^{2} / 2 \gamma_{b}^{2} \omega_{\beta \perp}^{2} \ll 1$, it follows from Eqs. (9) and (10) that $r_{b}<\lambda_{D \perp}$, corresponding to an emittance-dominated beam with near-parabolic density profile, $n^{0}(r)=\hat{n}\left(1-r^{2} / r_{b}^{2}\right)$, over the beam cross section. On the other hand, for $s_{b}=\hat{\omega}_{p b}^{2} / 2 \gamma_{b}^{2} \omega_{\beta \perp}^{2} \rightarrow 1-\epsilon$, with $\epsilon \rightarrow 0_{+}$, it follows from Eqs. (9) and (10) that $r_{b} \gg \lambda_{D \perp}$ and that $n^{0}(r)$ approaches the step-function density profile characteristic of space-charge-dominated beams with very low transverse emittance. These properties are illustrated in Fig. 1, where the normalized density profile $n^{0}(r) / \hat{n}$ calculated from Eq. (9) is plotted versus $r / r_{b}$ for several values of the dimensionless intensity parameter $s_{b}=\hat{\omega}_{p b}^{2} / 2 \gamma_{b}^{2} \omega_{\beta \perp}^{2}$ over the interval $0<s_{b}<1$.

The macroscopic fluid-Poisson equations (1)-(6) can be linearized for small-amplitude perturbations about the warm-fluid waterbag equilibrium described by Eqs. (7), (9), $\mathbf{V}_{\perp}^{0}(\mathbf{x})=0$, and $V_{z}^{0}=V_{b}=$ const. The resulting linearized equations then can be combined into a single eigenvalue equation ${ }^{26}$ for the perturbed electrostatic potential $\delta \phi(\mathrm{x}, t)$, allowing for arbitrary anisotropy in the perpendicular and parallel pressures, $P_{\perp}^{0}(r)$ and $P_{\|}^{0}(r)$. The details of this derivation will be presented elsewhere ${ }^{26}$.

Using a normal-mode approach, we express all perturbed quantities $\delta \phi(\mathbf{x}, t), \delta P_{\perp}(\mathbf{x}, t)$, $\delta P_{\|}(\mathrm{x}, t)$, and $\delta n(\mathrm{x}, t)$ as

$$
\delta \psi(\mathbf{x}, t)=\delta \hat{\psi}\left(\mathbf{x}_{\perp}\right) \exp \left(i k_{z} z-i \omega t\right)
$$

Here, $\delta \hat{\psi}\left(\mathrm{x}_{\perp}\right)$ is the perturbation amplitude, $k_{z}$ is the axial wavenumber of the perturbation, and $\omega$ is the complex oscillation frequency, with $\operatorname{Im} \omega>0$ corresponding to instability (temporal growth). We further introduce the definitions

$$
\Omega=\omega-k_{z} V_{b}, \quad \omega_{p b}^{2}(r)=\frac{4 \pi n^{0}(r) q^{2}}{\gamma_{b} m}, \quad v_{T z}^{2}=\frac{2 \hat{T}_{\|}}{\gamma_{b} m},
$$

where $\Omega$ is the Doppler-shifted oscillation frequency, $\omega_{p b}^{2}(r)$ is the local relativistic plasma 
frequency-squared, and $v_{T z}=$ const. is the effective axial thermal speed of the beam ions. After some algebraic manipulation and rearrangement of terms, we obtain ${ }^{26}$

$$
\begin{aligned}
\frac{\partial}{\partial \mathbf{x}_{\perp}} & \cdot\left\{\left[\Omega^{2}-\frac{\omega_{p b}^{2}(r)}{\gamma_{b}^{2}}\left(1+k_{z}^{2} \lambda_{D \perp}^{2}\right)-\frac{\frac{1}{2} k_{z}^{2} v_{T Z}^{2} \Omega^{2}}{\Omega^{2}-k_{z}^{2} v_{T Z}^{2}}\right] \frac{\partial}{\partial \mathbf{x}_{\perp}} \delta \hat{\phi}\right. \\
& +\lambda_{D \perp}^{2} \frac{\omega_{p b}^{2}(r)}{\gamma_{b}^{2}} \frac{\partial}{\partial \mathbf{x}_{\perp}} \nabla_{\perp}^{2} \delta \hat{\phi} \\
& \left.+\frac{1}{2} k_{z}^{2} \lambda_{D \perp}^{2} \frac{\partial}{\partial \mathbf{x}_{\perp}}\left[\frac{\omega_{p b}^{2}(r) / \gamma_{b}^{2}}{\Omega^{2}-k_{z}^{2} v_{T Z}^{2}}\left(\left[\omega_{p b}^{2}(r)+\frac{1}{2} k_{z}^{2} v_{T Z}^{2}\right] \delta \hat{\phi}-\frac{1}{2} v_{T Z}^{2} \nabla_{\perp}^{2} \delta \hat{\phi}\right)\right]\right\} \\
& -k_{z}^{2}\left\{\Omega^{2}-\frac{\omega_{p b}^{2}(r) \Omega^{2}}{\Omega^{2}-k_{z}^{2} v_{T Z}^{2}}-\frac{\frac{1}{2} k_{z}^{2} v_{T Z}^{2} \Omega^{2}}{\Omega^{2}-k_{z}^{2} v_{T Z}^{2}}\right\} \delta \hat{\phi}=0 .
\end{aligned}
$$

Equation (13) is the final eigenvalue equation for the potential eigenfunction $\delta \hat{\phi}\left(\mathbf{x}_{\perp}\right)$ and the eigenfrequency $\Omega=\omega-k_{z} V_{b}$. Equation (13) has been derived from Eqs. (1)-(6) for smallamplitude perturbations about the warm-fluid waterbag equilibrium described by Eqs. (7) and (9), and can be used to investigate detailed stability properties over a wide range of values of beam intensity and temperature anisotropy. Introducing cylindrical polar coordinates $(r, \theta)$, where $x=r \cos \theta$ and $y=r \sin \theta$, we represent $\delta \hat{\phi}\left(\mathbf{x}_{\perp}\right)=\delta \hat{\phi}(r, \theta)$. The eigenvalue equation (13) is to be solved for $\delta \hat{\phi}(r, \theta)$ and $\Omega$ subject to the requirements that $\delta \hat{\phi}(r, \theta)$ be regular at the origin $(r=0)$, and that

$$
\delta \hat{\phi}\left(r=r_{w}, \theta\right)=0
$$

The boundary condition in Eq. (14) assures that the perturbed tangential electric field components vanish at the perfectly conducting wall.

Anisotropy-driven instabilities are expected to be the strongest (largest growth rate) in the case of strong temperature anisotropy $\hat{T}_{\perp} \gg \hat{T}_{\|}$. In circumstances where the beam ions are cold in the propagation direction, the eigenvalue equation (13) simplifies considerably. Therefore, in the remainder of this article we set $\hat{T}_{\|}=0\left(v_{T z}^{2}=0\right)$ in Eq. (13), and further assume axisymmetric perturbations with $(\partial / \partial \theta) \delta \hat{\phi}=0$. The eigenvalue equation (13) then reduces to ${ }^{26}$ 


$$
\begin{aligned}
& \frac{1}{r} \frac{\partial}{\partial r} r\left\{\left[\Omega^{2}-\frac{\omega_{p b}^{2}(r)}{\gamma_{b}^{2}}\left(1+k_{z}^{2} \lambda_{D \perp}^{2}\right)\right] \frac{\partial}{\partial r} \delta \hat{\phi}(r)\right. \\
& +\lambda_{D \perp}^{2} \frac{\omega_{p b}^{2}(r)}{\gamma_{b}^{2}}\left(\frac{1}{r} \frac{\partial}{\partial r} r \frac{\partial}{\partial r}-\frac{1}{r^{2}}\right) \frac{\partial}{\partial r} \delta \hat{\phi}(r) \\
& \left.+\frac{1}{2} k_{z}^{2} \lambda_{D \perp}^{2} \frac{\partial}{\partial r}\left(\frac{\omega_{p b}^{4}(r) / \gamma_{b}^{2}}{\Omega^{2}} \delta \hat{\phi}(r)\right)\right\} \\
& -k_{z}^{2}\left[\Omega^{2}-\omega_{p b}^{2}(r)\right] \delta \hat{\phi}(r)=0 .
\end{aligned}
$$

For $k_{z}=0$ and $\lambda_{D \perp} \neq 0$, it is found ${ }^{26}$ that Eq. (15) gives purely stable oscillations with $\operatorname{Im} \hat{\Omega}=0$. On the other hand, as $k_{z} r_{b}$ is increased to sufficiently large values, the temperature anisotropy $\left(\hat{T}_{\perp} \propto \lambda_{D \perp}^{2} \neq 0\right.$, and $\left.\hat{T}_{\|}=0\right)$ provides the free energy to drive an instability ${ }^{26}$ at moderate values of beam intensity.

For the equilibrium density profile $n^{0}(r)$ specified by Eq. (9), the eigenvalue equation (15) must be solved numerically for the (generally) complex eigenfunction $\delta \hat{\phi}=\delta \hat{\phi}_{r}+i \delta \hat{\phi}_{i}$ and complex eigenfrequency $\Omega=\Omega_{r}+i \Omega_{i}=\omega_{r}+i \omega_{i}-k_{z} V_{b}$, subject to the boundary condition at $r=r_{w}$ in Eq. (14). A detailed investigation of Eq. (15) for a wide range of system parameters will be presented elsewhere ${ }^{26}$, and we summarize here several of the key results. First, the dimensionless parameters characterizing Eq. (15) are

$$
k_{z} r_{b}, \quad \Gamma_{D} \equiv \frac{\lambda_{D \perp}}{r_{b}}, \quad s_{b} \equiv \frac{\omega_{p b}^{2}}{2 \gamma_{b}^{2} \omega_{\beta \perp}^{2}}, \quad \frac{r_{b}}{r_{w}},
$$

where $s_{b}$ and $\Gamma_{D}$ are related by Eq. (10). Second, it is convenient to introduce the characteristic measures of the depressed and undepressed single-particle transverse oscillation frequencies, $\nu$ and $\nu_{0}$, defined by

$$
\nu^{2} \equiv \omega_{\beta \perp}^{2}-\hat{\omega}_{p b}^{2} / 2 \gamma_{b}^{2}, \quad \nu_{0}^{2} \equiv \omega_{\beta \perp}^{2},
$$

and the effective tune depression, $\nu / \nu_{0}$, where

$$
\frac{\nu^{2}}{\nu_{0}^{2}}=1-\frac{\hat{\omega}_{p b}^{2}}{2 \gamma_{b}^{2} \omega_{\beta \perp}^{2}}=1-s_{b} .
$$

Finally, in Eq. (15) there is a clear demarcation between the vacuum region where $\omega_{p b}^{2}(r)=0$ $\left(r_{b}<r \leq r_{w}\right)$, and the beam interior $\left(0 \leq r<r_{b}\right)$. For $s_{b} \neq 1$ and $\Gamma_{D} \neq 0$, the equilibrium 
density profile $n^{0}(r)$ in Eq. (9) approaches zero continuously at $r=r_{b}$ (see Fig. 1), and it is readily shown ${ }^{26}$ from Eq. (15) that the appropriate boundary conditions at the beam surface $\left(r=r_{b}\right)$ correspond to continuity of $\delta \hat{\phi}(r)$,

$$
\begin{gathered}
\operatorname{Re}\left[\delta \hat{\phi}^{I}\left(r=r_{b}\right)\right]=\operatorname{Re}\left[\delta \hat{\phi}^{I I}\left(r=r_{b}\right)\right], \\
\operatorname{Im}\left[\delta \hat{\phi}^{I}\left(r=r_{b}\right)\right]=\operatorname{Im}\left[\delta \hat{\phi}^{I I}\left(r=r_{b}\right)\right],
\end{gathered}
$$

and continuity of $(\partial / \partial r) \delta \hat{\phi}(r)$

$$
\begin{aligned}
\operatorname{Re}\left[\frac{\partial}{\partial r} \delta \hat{\phi}^{I}(r)\right]_{r=r_{b}} & =\operatorname{Re}\left[\frac{\partial}{\partial r} \delta \hat{\phi}^{I I}(r)\right]_{r=r_{b}}, \\
\operatorname{Im}\left[\frac{\partial}{\partial r} \delta \hat{\phi}^{I}(r)\right]_{r=r_{b}} & =\operatorname{Im}\left[\frac{\partial}{\partial r} \delta \hat{\phi}^{I I}(r)\right]_{r=r_{b}},
\end{aligned}
$$

where Region I corresponds to the beam interior $\left(0 \leq r<r_{b}\right)$, and Region II corresponds to the vacuum region $\left(r_{b}<r \leq r_{w}\right)$.

For $k_{z}=0$. it is readily shown from Eq. (15) that the radial electric field perturbation, $\delta \hat{E}_{r}=-(\partial / \partial r) \delta \hat{\phi}$, is equal to zero in the vacuum region, i.e., $\delta \hat{E}_{r}=0$ for $r_{b}<r \leq r_{w}$. For $k_{z}=0$, Eq. (15) has been solved ${ }^{26}$ over the interval $0 \leq r<r_{b}$ for $\delta \hat{E}_{r}(r)$ and $\Omega$ subject to the requirement that $\left[\delta \hat{E}_{r}\right]_{r=r_{b}}=0$ using two approaches: (a) direct numerical integration of Eq. (15) using a shooting method to determine both the eigenfunctions and eigenvalues, and (b) a matrix-dispersion-equation technique that expands Eq. (15) in a complete set of basis functions that satisfy a priori the boundary condition $\left[\delta \hat{E}_{r}\right]_{r=r_{b}}=0$. The results using both techniques are in excellent agreement ${ }^{26}$. For $k_{z}=0$, the solutions to Eq. (15) correspond to a discrete set of stable oscillations $\left\{\omega_{n}\right\}$ with radial mode numbers $n=1,2,3, \ldots$, and $\operatorname{Im} \omega_{n}=0$. Typical numerical results are shown in Fig. 2 where $\operatorname{Re}\left[\omega_{n} / \nu_{0}\right]$ is plotted versus $\nu / \nu_{0}$ in Fig. 2(a) for effective tune depressions ranging from $\nu / \nu_{0}=0\left(s_{b}=1\right)$ to $\nu / \nu_{0}=1$ $\left(s_{b}=0\right)$. For each value of $n$, note that the $\nu / \nu_{0}=0\left(\hat{\omega}_{p b}^{2} / 2 \gamma_{b}^{2} \omega_{\beta \perp}^{2}=1\right)$ intercept in Fig. 2(a) corresponds to the single frequency $R e \omega_{n}=\sqrt{2} \nu_{0}=\sqrt{2} \omega_{\beta \perp}=\hat{\omega}_{p b} / \gamma_{b}$ in the limit of high beam intensity. On the other hand, as $\nu / \nu_{0}=\left(1-s_{b}\right)^{1 / 2}$ is increased (decreasing beam intensity $\left.s_{b}=\hat{\omega}_{p b}^{2} / 2 \gamma_{b}^{2} \omega_{\beta \perp}^{2}\right)$, it is evident from Fig. 2(a) that there is a discrete spectrum 
of stable oscillations with frequencies $\left\{\omega_{n}\right\}$ that increase as $\nu / \nu_{0}$ and $n$ are increased. The general features of the solutions for $\left\{\omega_{n}\right\}$ presented in Fig. 2(a) for $k_{z}=0$ perturbations about a warm-fluid waterbag equilibrium are qualitatively similar to those for a warm-fluid Kapchinskij-Vladimirskij (KV) equilibrium ${ }^{24}$, although the precise values of $\left\{\omega_{n}\right\}$ differ as $\nu / \nu_{0}$ is increased. For completeness, shown in Fig. 2(b) are plots of the eigenfunction $\delta \hat{E}(r)$ versus $r / r_{b}$ for radial mode numbers $n=1,2,3$ obtained numerically from Eq. (15) for $s_{b}=0.36$ and $\nu / \nu_{0}=0.8$. Note from Fig. $2(\mathrm{~b})$ that the number of radial oscillations of $\delta \hat{E}(r)$ increase as the mode number $n$ is increased. Moreover, for specified mode number $n$, the number of zeros of $\delta \hat{E}(r)$ in the interval $0 \leq r \leq r_{b}$ is equal to $n+1$.

For $k_{z} \neq 0$ and $\Gamma_{D}=\lambda_{D \perp} / r_{b} \neq 0$, the eigenvalue equation (15), valid for $\hat{T}_{\|}=0$, generally supports unstable solutions $(\operatorname{Im} \omega>0)$ provided $k_{z} r_{b}$ is sufficiently large and the normalized beam intensity $s_{b}$ is sufficiently below the space-charge limit ${ }^{26}$. The eigenvalue equation (15) is a linear fourth-order ordinary differential equation for $\delta \hat{\phi}(r)$. At $r=0$, several of the coefficients are singular. At $r=r_{b}$, the beam edge, the coefficient multiplying the highest-derivative term vanishes, causing a boundary layer. Since standard numerical integration techniques are not applicable, we instead expand the solution near $r=0$ in a Froebenius series. Using this analytical expansion near the origin where it is sufficiently accurate, we begin by numerically integrating from very near $r=0$ out to the beam edge at $r=r_{b}$, and then back to the origin.

The linearity of Eq. (15) implies that the solution for $\delta \hat{\phi}(r)$ is arbitrary up to a constant, multiplicative, complex factor. This freedom can be used in the unstable case to make the matching of real and imaginary parts at the beam edge simpler. For present purposes, we choose the initial amplitude and phase at $r=0$ such that $\operatorname{Re}[\hat{\phi}(r=0)]=1$ and $\operatorname{Im}[\delta \hat{\phi}(r=0)]=0$. In the unstable case, integrating away from $r=0$, the eigenfunction generally develops an imaginary component with $\operatorname{Im}[\delta \hat{\phi}(r)] \neq 0$. Of course at the beam edge $\left(r=r_{b}\right)$, there are four conditions to satisfy, corresponding to Eqs. (19) and (20). We can automatically satisfy the matching conditions in Eq. (19) by appropriate choice of complex phase factor for the solution for $\delta \hat{\phi}(r)$ in the vacuum region $\left(r_{b}<r \leq r_{w}\right)$. In general, 
however, neither of the matching conditions on $\partial \delta \hat{\phi} / \partial r$ at $r=r_{b}$ in Eq. (20) will be satisfied unless the complex eigenfrequency $\Omega=\Omega_{r}+i \Omega_{i}$ occurring in Eq. (15) is correctly chosen, which corresponds to the desired dispersion relation. Therefore, in the present shooting method, Eq. (15) is repeatedly integrated, and the value of $\Omega_{r}+i \Omega_{i}$ adjusted until the matching conditions in Eq. (19) are satisfied, thereby determining the eigenfrequency.

Using this method, Eq. (15) has been solved numerically subject to the boundary conditions in Eqs. (14), (19) and (20), and the complex eigenfrequency $\Omega=\Omega_{r}+i \Omega_{i}$ and eigenfunction $\delta \hat{\phi}(r)$ have been determined self-consistently over a wide range of system parameters corresponding to normalized beam intensity, $s_{b}=\hat{\omega}_{p b}^{2} / 2 \gamma_{b}^{2} \omega_{\beta \perp}^{2}$, tune depression, $\nu / \nu_{0}=\left(1-s_{b}\right)^{1 / 2}$, transverse Debye length, $\Gamma_{D}=\lambda_{D \perp} / r_{b}$, and axial wavenumber $k_{z} r_{b}$. Here, keep in mind that $s_{b}$ and $\Gamma_{D}$ are related by Eq. (10), so that very high beam intensity $\left(s_{b} \rightarrow 1\right)$ corresponds to $\Gamma_{D} \ll 1$, and low beam intensity $\left(s_{b} \ll 1\right)$ corresponds to $\Gamma_{D} \gg 1$ (see Fig. 1). Because $\hat{T}_{\|}=0$ is assumed in the present analysis, the term proportional to $k_{z}^{2} \lambda_{D \perp}^{2} \neq 0$ in Eq. (15) provides the free energy to drive instability associated with temperature anisotropy $\left(\hat{T}_{\perp}>\hat{T}_{\|}\right)$.

For $k_{z} \neq 0$, typical numerical results obtained from Eq. (15), are illustrated in Figs. $3-5$ for a mildly relativistic beam with $\gamma_{b}=1.02$ and $r_{w} / r_{b}=2$. As a general remark, beams which are cold in the transverse direction oscillate stably at all values of axial wavelength. Beams with intermediate transverse temperatures are unstable for all wavenumbers $k_{z} r_{b}$ larger than a critical value. Finally, beams which are sufficiently hot in the transverse direction have a finite instability bandwidth in $k_{z} r_{b}$ : short wavelengths and long wavelengths are stable, while wavelengths in a range about $k_{z} r_{b} \Gamma_{D} \sim 1$ are unstable. That is, the growth rate of sufficiently temperature-dominated beams turns over and approaches zero at large values of $k_{z} r_{b}$. For the choice of waterbag equilibrium considered here with $\hat{T}_{\|}=0$, the onset of instability occurs for $\Gamma_{D}>\Gamma_{D}^{*}=0.364, s_{b}<s_{b}^{*}=0.750$, and $\nu / \nu_{0}>\nu^{*} / \nu_{0}=0.500$, which are equivalent conditions. For increasing values of $\Gamma_{D}$ relative to $\Gamma_{D}^{*}$, the instability bandwidth first increases, encompassing both higher and lower axial wavenumbers. For even 
warmer beams, however, high values of $k_{z} r_{b}$ become stabilized, while the region of instability continues to shift to smaller axial wavenumbers.

Figure 3 shows typical numerical results for the choice of system parameters $\Gamma_{D}=0.360$ $\left(s_{b}=0.755\right)$, corresponding to stable oscillations with $\operatorname{Im} \Omega=\operatorname{Im} \omega=0$. Plotted in Fig. 3(a) is $\operatorname{Re}\left(\omega-k_{z} V_{b}\right) / \nu_{0}$ versus $k_{z} r_{b}$ for the $n=1$ eigenmode, whereas Fig. 3(b) shows the corresponding eigenfunction $\delta \hat{\phi}(r)$ (assumed real) plotted versus $r / r_{b}$ for several values of $k_{z} r_{b}$. The high-frequency (upper) branch in Fig. 3(b) corresponds to the familiar plasma oscillation branch considered in Fig. 2, extended to non-zero values of $k_{z} r_{b}$. The lower (slow-wave) branch in Fig. 3(a) starts at zero frequency for $k_{z}=0$, and $\operatorname{Re}\left(\omega-k_{z} V_{b}\right)$ increases linearly with $k_{z} r_{b}$ for $k_{z} r_{b}<1$, and then asymptotes at $\operatorname{Re}\left(\omega-k_{z} V_{b}\right) \simeq 1.03 \nu_{0}$ for $k_{z} r_{b} \gg 1$. On the other hand, the upper (high-frequency) branch in Fig. 3(a) asymptotes at $\operatorname{Re}\left(\omega-k_{z} V_{b}\right) \simeq 1.3 \nu_{0}$ for $k_{z} r_{b} \gg 1$. From Fig. 3(b), as expected, as $k_{z} r_{b}$ is increased, the eigenfunction $\delta \hat{\phi}(r)$ extends radially well into the vacuum region, with $\delta \hat{\phi}\left(r=r_{w}\right)=0$.

For $\Gamma_{D}>\Gamma_{D}^{*}$, the two branches in Fig. 3 coalesce as $k_{z} r_{b}$ is increased beyond some critical value $k_{z}^{*}\left(\Gamma_{D}\right) r_{b}$. Typical numerical results in this case are illustrated in Fig. 4 for the choice of system parameters $\Gamma_{D}=0.509\left(s_{b}=0.55\right)$. From Fig. 4 , for $0 \leq k_{z} r_{b}<$ $k_{z}^{*} r_{b}=0.968$, the eigenvalue equation supports two real oscillatory solutions with $\operatorname{Im} \omega=0$. For $k_{b} r_{b}>k_{z}^{*} r_{b}=0.968$, however, the two modes coalesce and have the same value of $\operatorname{Re}\left(\omega-k_{z} V_{b}\right)$, and complex conjugate values of Im $\omega$ (one mode is damped, and the other is growing). The normalized growth rate $\operatorname{Im} \omega / \nu_{0}$ of the unstable branch is plotted versus $k_{z} r_{b}$ in Fig. 4(a), and increases from $\operatorname{Im} \omega=0$ at $k_{z} r_{b}=k_{z}^{*} r_{b}=0.968$, to $\operatorname{Im} \omega \simeq 0.4 \nu_{0}$ for $k_{z} r_{b} \gg 1$. Consistent with Fig. 4(a), the corresponding eigenfunction plots of $\operatorname{Re}[\delta \hat{\phi}(r)]$ and $\operatorname{Im}[\delta \hat{\phi}(r)]$ versus $r / r_{b}$ are presented in Fig. $4(\mathrm{~b})$ for $k_{b} r_{b}=4$, corresponding to instability. For moderately low values of $k_{z} r_{b}$, the eigenfunction for the unstable mode has the distinctive $n=1$ mode structure illustrated in Fig. 4(b) for $k_{z} r_{b}=4$. As $k_{z} r_{b}$ is increased, however, the real part of the eigenfunction, $\operatorname{Re}[\delta \hat{\phi}(r)]$, changes continuously from an $n=1$ to an $n=2$ mode structure ${ }^{26}$. For very large $k_{z} r_{b}>10$, the boundary layer at $r=r_{b}$ becomes very sharp, with large changes in $\operatorname{Re}[\delta \hat{\phi}(r)]$ over a very short radial scale ${ }^{26}$. The radial mode 
number, however, does not appear to change from $n=2$. Of course, perturbations with such large values of $k_{z} r_{b}$ are of limited practical interest because the modes would be stabilized $(I m \omega=0)$ at short axial wavelengths by finite $\hat{T}_{\|} \neq 0$ effects in an analysis of the more complete eigenvalue equation (13).

For completeness, shown in Fig. 5 are plots of the normalized growth rate $\operatorname{Im} \omega / \nu_{0}$ versus $k_{z} r_{b}$ obtained numerically from Eqs. (14), (15), (19), and (20) for several values of $\Gamma_{D}>\Gamma_{D}^{*}$ and $s_{b}<s_{b}^{*}$. Note from Fig. 5 that critical value of $k_{z} r_{b}$ for onset of instability increases as $\Gamma_{D}$ is increased $\left(s_{b}\right.$ is decreased), and that the maximum normalized growth rate $(I \operatorname{m\omega })_{\max } / \nu_{0}$ increases as $\Gamma_{D}$ is increased $\left(s_{b}\right.$ is decreased). For sufficiently large value of $\Gamma_{D}$ (large enough transverse emittance), we also note from Fig. 5 that the instability has a finite bandwidth in $k_{z} r_{b}$, whereas for smaller values of $\Gamma_{D}$, the maximum growth rate occurs for $k_{z} r_{b} \gg 1$. For $\hat{T}_{\|} \neq 0$ (but $\hat{T}_{\|}<\hat{T}_{\perp}$ ), it is expected that the more complete eigenvalue equation (13) will always give a finite instability bandwidth in $k_{z} r_{b}$.

To summarize, for $k_{z}=0$, the analysis of the eigenvalue equation (15) leads to a discrete spectrum $\left\{\omega_{n}\right\}$ of stable oscillations with $\operatorname{Im} \omega_{n}=0$, where $n$ is the radial mode number. On the other hand, for sufficiently large values of $k_{z} r_{b}$, where $r_{b}$ is the beam radius, the analysis of the eigenvalue equation leads to an anisotropy-driven instability $(\operatorname{Im\omega }>0)$ provided the normalized Debye length $\left(\Gamma_{D}=\lambda_{D \perp} / r_{b}\right)$ is sufficiently large and the normalized beam intensity $\left(s_{b}=\hat{\omega}_{p b}^{2} / 2 \gamma_{b}^{2} \omega_{\beta \perp}^{2}\right)$ is sufficiently below the space-charge limit. Depending on system parameters, the growth rate can be a substantial fraction of the focusing frequency $\omega_{\beta \perp}$. As a general remark, application of a warm-fluid model to describe the equilibrium and stability properties of intense charged particle beams appears to be a remarkably robust and simple approach, both for the case of stable high-frequency collective oscillations ${ }^{24}$, as well as the unstable case considered here, where the instability is driven by gross macroscopic properties of the beam equilibrium (pressure anisotropy). 


\section{ACKNOWLEDGMENTS}

This research was supported by the U.S. Department of Energy. 


\section{REFERENCES}

${ }^{1}$ R. C. Davidson, Physics of Nonneutral Plasmas (Addison-Wesley, Reading, MA, 1990), and references therein.

${ }^{2}$ T. P. Wangler, Principles of RF Linear Accelerators (Wiley, New York, 1998).

${ }^{3}$ M. Reiser, Theory and Design of Charged Particle Beams (Wiley, New York, 1994).

${ }^{4}$ I. Kapchinskij and V. Vladimirskij, in Proceedings of the International Conference on High Energy Accelerators and Instrumentation (CERN Scientific Information Service, Geneva, 1959), p. 274.

${ }^{5}$ R. L. Gluckstern, in Proceedings of the 1970 Proton Linear Accelerator Conference, Batavia, IL, 1971, edited by M. R. Tracy (National Accelerator Laboratory, Batavia, IL, 1971), p. 811.

${ }^{6}$ T. -S. Wang and L. Smith, Part. Accel. 12, 247 (1982).

${ }^{7}$ I. Hofmann, L. J. Laslett, L. Smith and I. Haber, Part. Accel. 13, 145 (1983).

${ }^{8}$ I. Hofmann and J. Struckmeier, Part. Accel. 21, 69 (1987).

${ }^{9}$ R. L. Gluckstern, W. -H. Cheng and H. Yee, Phys. Rev. Lett. 75, 2835 (1995).

${ }^{10}$ C. Chen, R. Pakter and R. C. Davidson, Phys. Rev. Lett. 79, 225 (1997).

${ }^{11}$ J. Struckmeier and I. Hofmann, Part. Accel. 39, 219 (1992).

12 N. Brown and M. Reiser, Phys. Plasmas 2, 965 (1995).

${ }^{13}$ R. C. Davidson and C. Chen, Part. Accel. 59, 175 (1998).

${ }^{14}$ R. C. Davidson, Phys. Rev. Lett. 81, 991 (1998).

${ }^{15}$ R. C. Davidson, H. Qin and P. J. Channell, Phys. Rev. Special Topics on Accelerators and Beams 2, 074401 (1999). 
${ }^{16}$ R. C. Davidson and H. Qin, Phys. Rev. Special Topics on Accelerators and Beams 2, $114401(1999)$.

${ }^{17}$ See, e.g., Chaps. 2, 4, 9 and 10 of Ref. 1.

${ }^{18}$ S. M. Lund, D. A. Callahan, A. Friedman, D. P. Grote, I. Haber and T. -S. Wang, Proceedings of the 19th International Linac Conference (Chicago, IL, 1998).

${ }^{19}$ I. Haber, D. A. Callahan, A. Friedman, D. P. Grote, S. M. Lund and T. -S. Wang, Nucl. Instrum. Methods Phys. Res. (in press).

${ }^{20}$ I. Haber, D. A. Callahan, A. Friedman, D. P. Grote and A. B. Langdon, J. Fusion Eng. Design 32, 159 (1996).

${ }^{21}$ Reference 1 presents a general derivation of the macroscopic fluid Maxwell equations from the Vlasov-Maxwell equations on pages 22-26. Several aspects of cold-fluid equilibrium and stability properties of nonneutral beam-plasma systems are described on pages 240276 of Ref 1.

${ }^{22}$ R. C. Davidson, P. Stoltz and C. Chen, Phys. Plasmas 4, 3710 (1997).

${ }^{23}$ I. Hofmann, Phys. Rev. E57, 4713 (1998).

${ }^{24}$ S. M. Lund and R. C. Davidson, Phys. Plasmas 5, 3028 (1998).

${ }^{25}$ C. Chen and R. Pakter, Phys. Plasmas 7, in press (2000).

${ }^{26}$ R. C. Davidson and S. Strasburg, "Warm-Fluid Stability Properties of Intense Nonneutral Charged Particle Beams with Pressure Anisotropy," submitted for publication (2000). 


\section{FIGURES}

FIG. 1. Plot of $n^{0}(r) / \hat{n}$ versus $r / r_{b}$ calculated from Eqs. (9) and (10) for several values of $s_{b}=\hat{\omega}_{p b}^{2} / 2 \gamma_{b}^{2} \omega_{\beta \perp}^{2}$.

FIG. 2. Plots of (a) Re $\omega_{n} / \nu_{0}$ versus $\nu / \nu_{0}$ for radial mode numbers $n=1, \cdots 4$, and (b) $\delta \hat{E}_{r}=-(\partial / \partial r) \delta \hat{\phi}$ versus $r / r_{b}$ for $n=1,2,3$, for $s_{b}=0.36$ and $\nu / \nu_{0}=0.8$, obtained numerically from the eigenvalue equation (15) for $k_{z}=0$.

FIG. 3. Plots of (a) $\operatorname{Re}\left(\omega-k_{z} V_{b}\right) / \nu_{0}$ versus $k_{z} r_{b}$, and (b) $\operatorname{Re}[\delta \hat{\phi}(r)]$ versus $r / r_{b}$ for several values of $k_{z} r_{b}$, obtained numerically from Eq. (15) for $\Gamma_{D}=0.360\left(s_{b}=0.755\right)$.

FIG. 4. Plots of (a) $\operatorname{Re}\left(\omega-k_{z} V_{b}\right) / \nu_{0}$ and $\operatorname{Im} \omega / \nu_{0}$ versus $k_{z} r_{b}$, and (b) $\operatorname{Re}[\delta \hat{\phi}(r)]$ and $\operatorname{Im}[\delta \phi(\hat{r})]$ versus $r / r_{b}$ for $k_{z} r_{b}=4$, obtained numerically from Eq. (15) for $\Gamma_{D}=0.509\left(s_{b}=0.55\right)$.

FIG. 5. Plots of $I m \omega / \nu_{0}$ versus $k_{z} r_{b}$ obtained numerically from Eq. (15) for several values of $\Gamma_{D}>\Gamma_{D}^{*}=0.364$ 


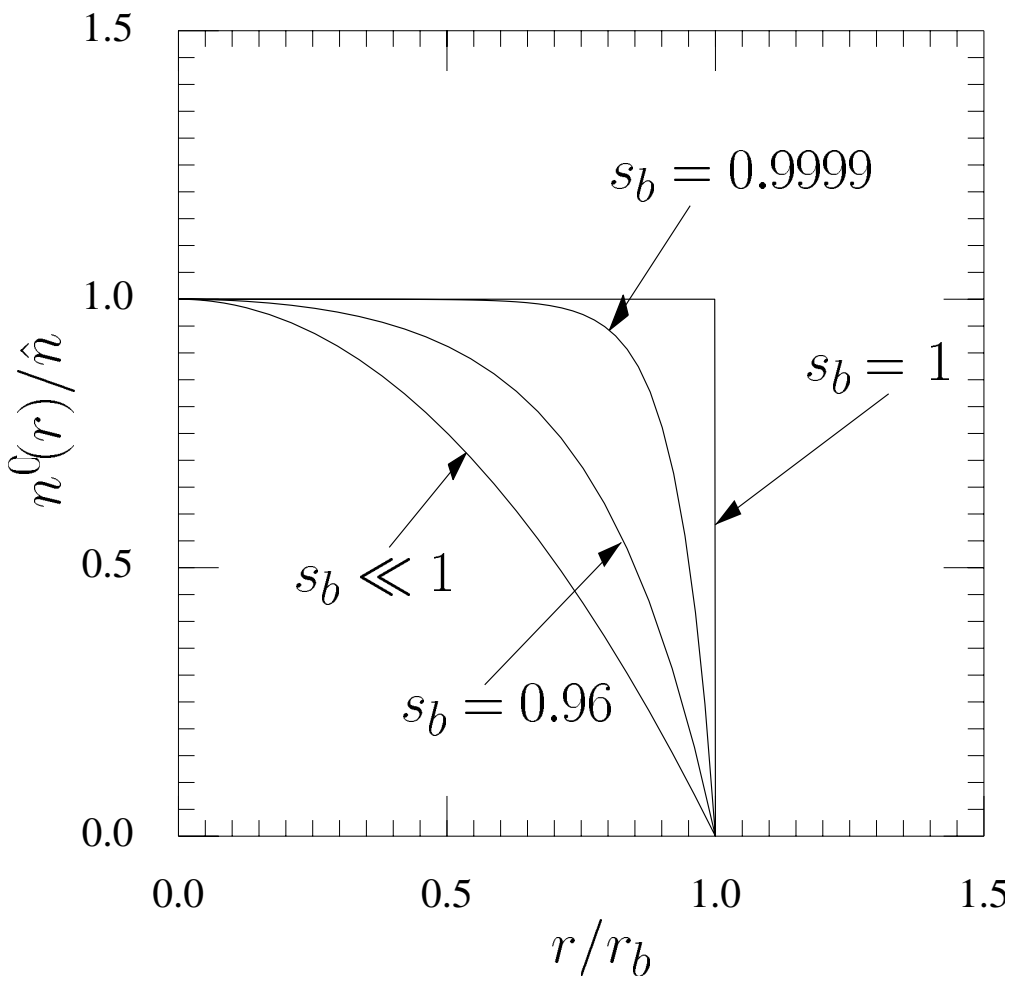

FIG. 1. Plot of $n^{0}(r) / \hat{n}$ versus $r / r_{b}$ calculated from Eqs. (9) and (10) for several values of $s_{b}=\hat{\omega}_{p b}^{2} / 2 \gamma_{b}^{2} \omega_{\beta \perp}^{2}$. 

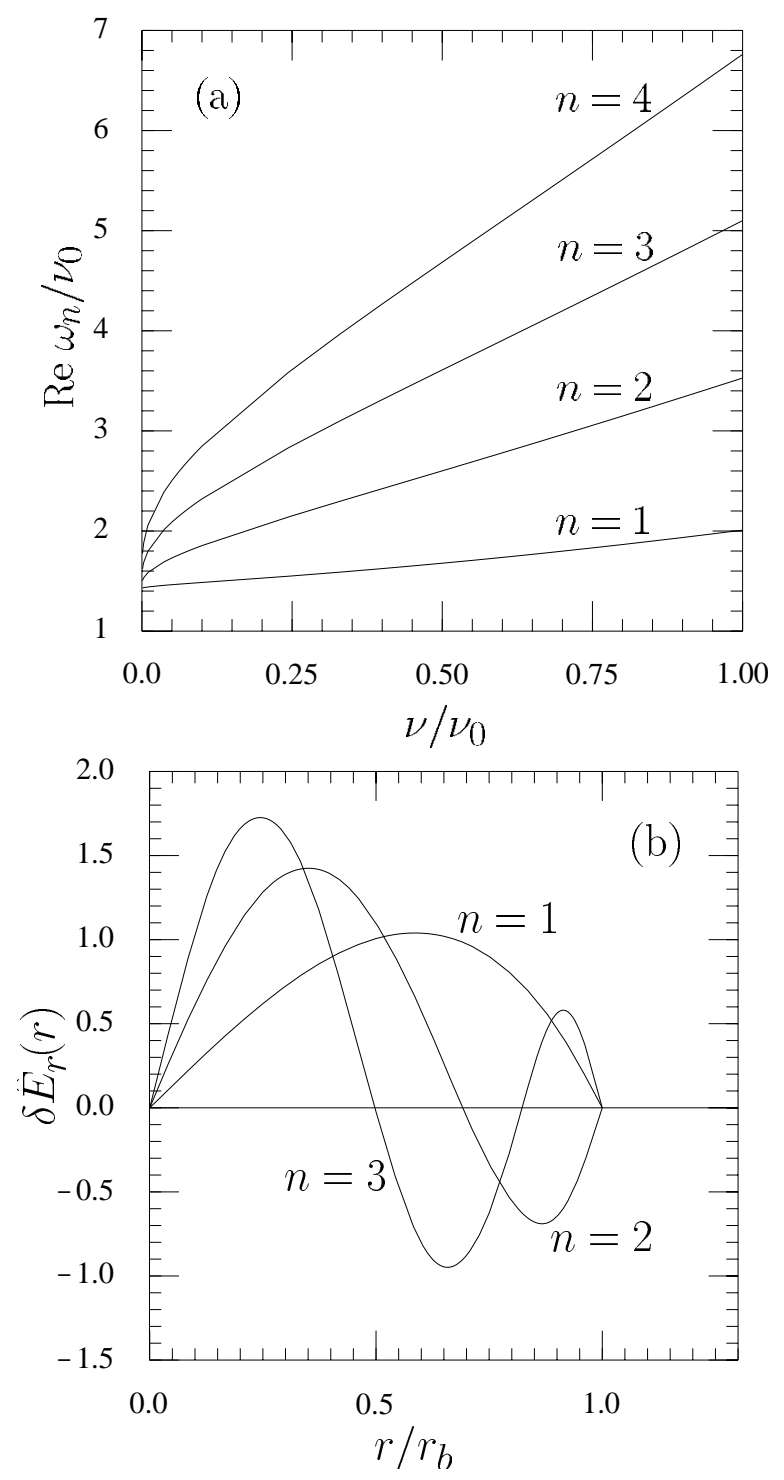

FIG. 2. Plots of (a) $R e \omega_{n} / \nu_{0}$ versus $\nu / \nu_{0}$ for radial mode numbers $n=$ $1, \cdots 4$, and (b) $\delta \hat{E}_{r}=-(\partial / \partial r) \delta \hat{\phi}$ versus $r / r_{b}$ for $n=1,2,3$, for $s_{b}=0.36$ and $\nu / \nu_{0}=0.8$, obtained numerically from the eigenvalue equation (15) for $k_{z}=0$. 

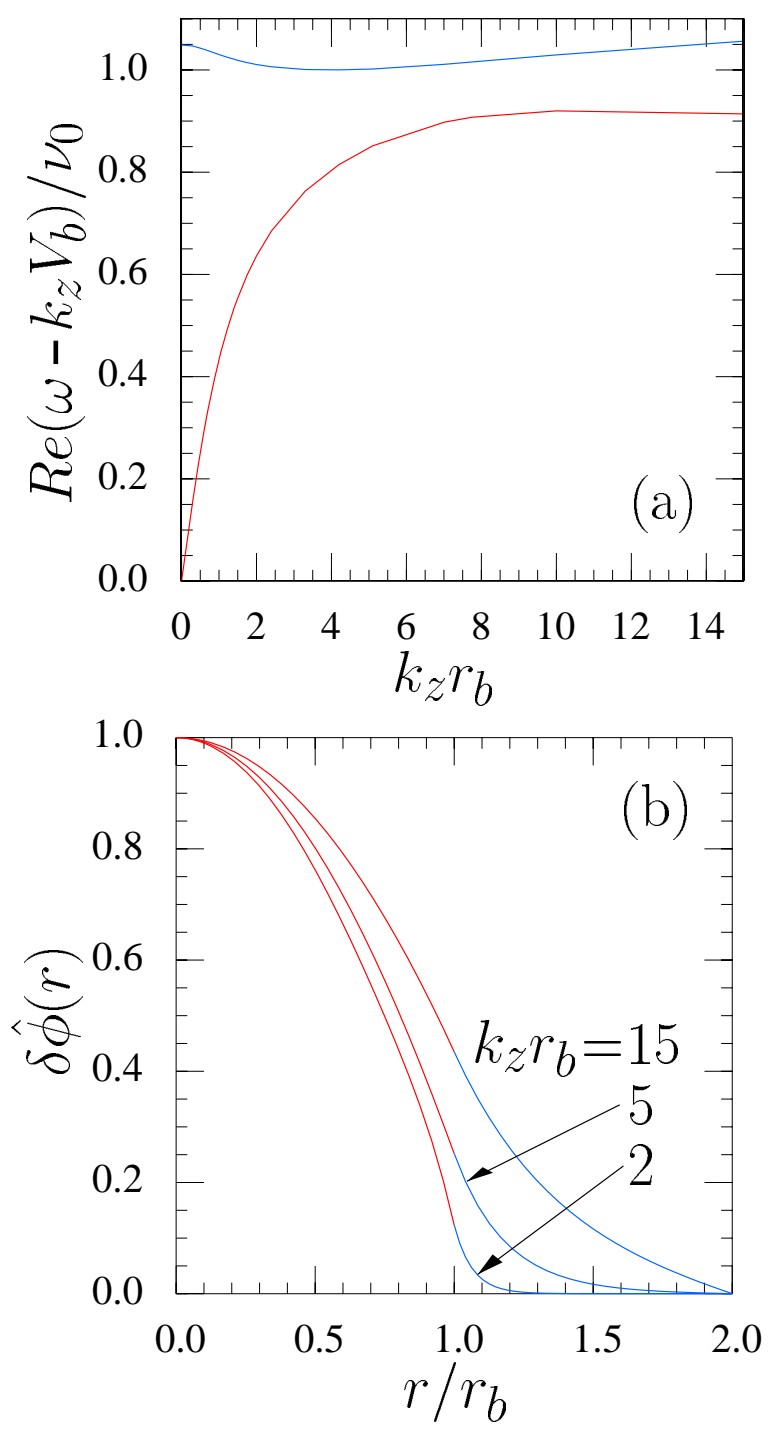

FIG. 3. Plots of (a) $\operatorname{Re}\left(\omega-k_{z} V_{b}\right) / \nu_{0}$ versus $k_{z} r_{b}$, and (b) $\operatorname{Re}[\delta \hat{\phi}(r)]$ versus $r / r_{b}$ for several values of $k_{z} r_{b}$, obtained numerically from Eq. (15) for $\Gamma_{D}=$ $0.360\left(s_{b}=0.755\right)$. 

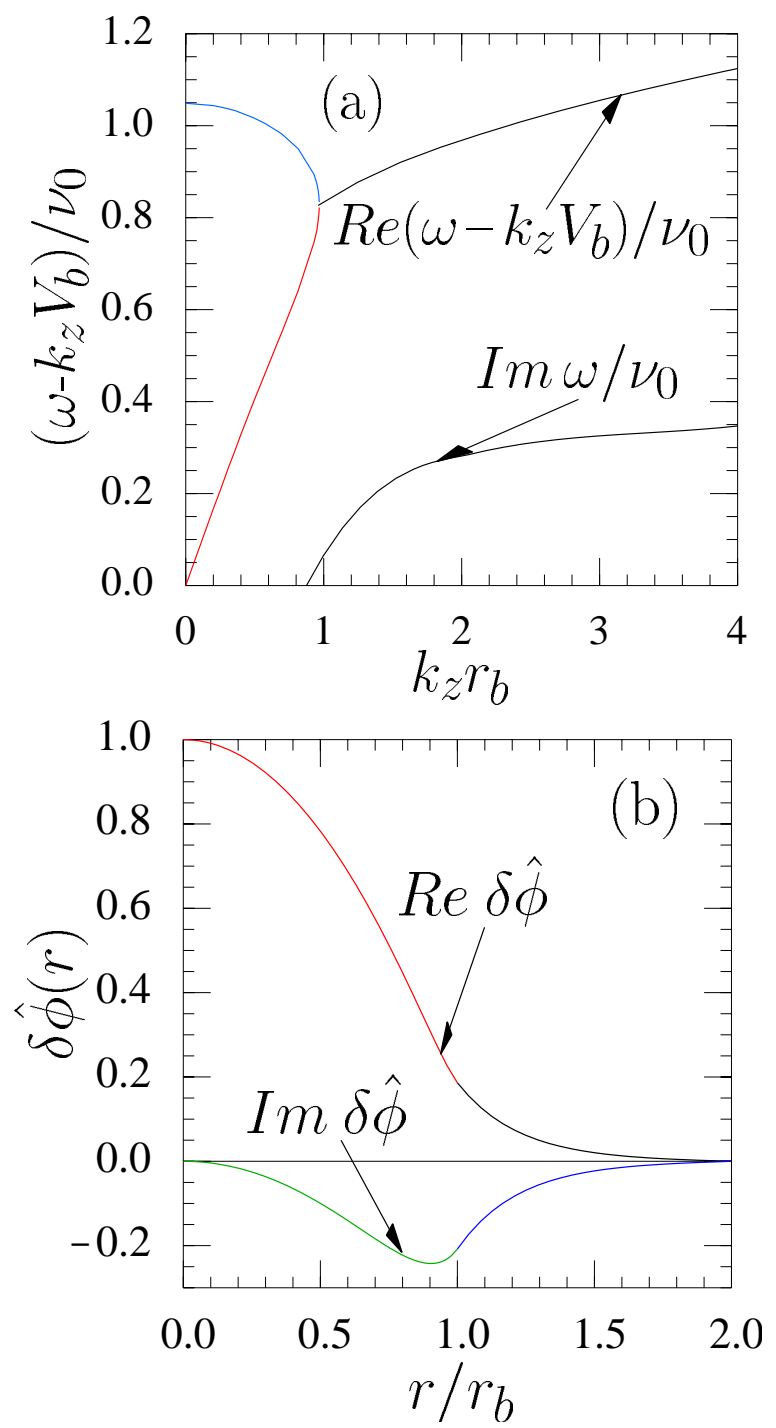

FIG. 4. Plots of (a) $\operatorname{Re}\left(\omega-k_{z} V_{b}\right) / \nu_{0}$ and $\operatorname{Im} \omega / \nu_{0}$ versus $k_{z} r_{b}$, and (b) $\operatorname{Re}[\delta \hat{\phi}(r)]$ and $\operatorname{Im}[\delta \phi(\hat{r})]$ versus $r / r_{b}$ for $k_{z} r_{b}=4$, obtained numerically from Eq. (15) for $\Gamma_{D}=0.509\left(s_{b}=0.55\right)$. 


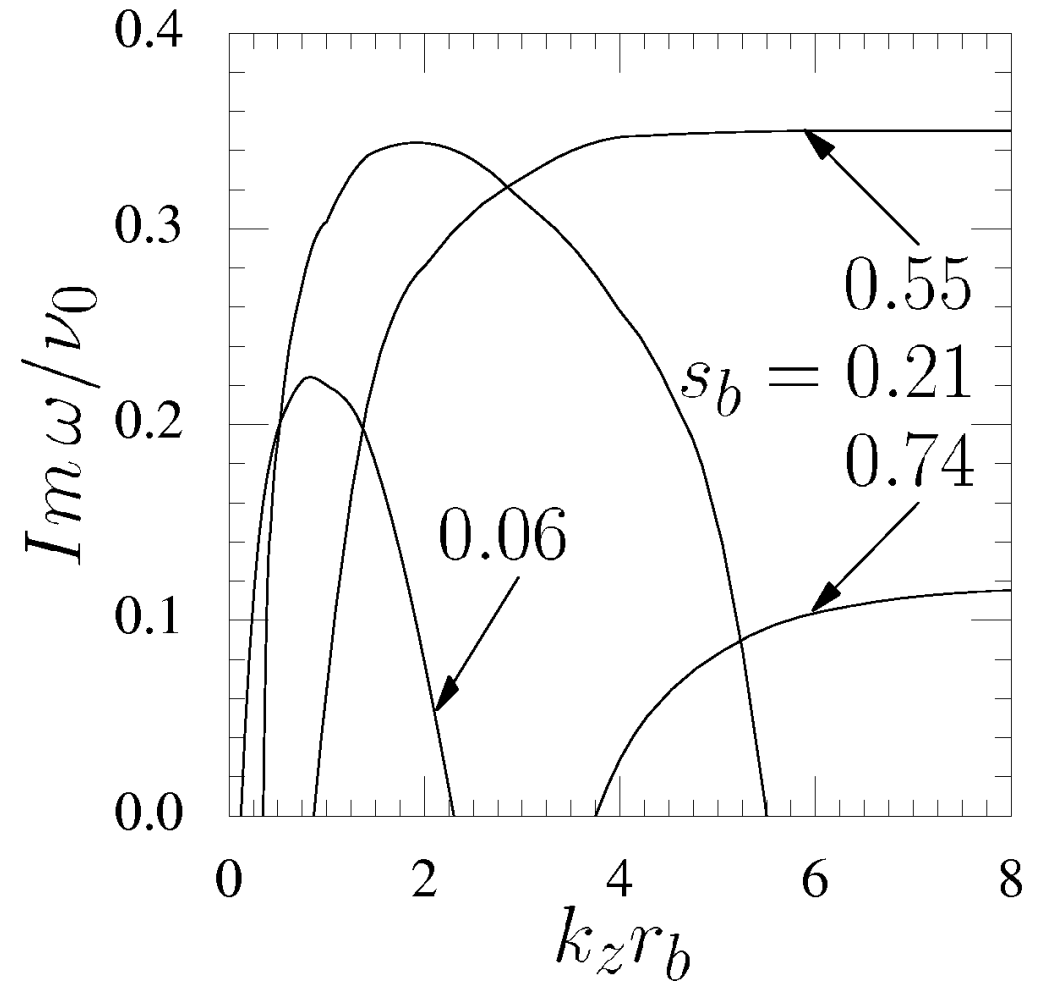

FIG. 5. Plots of $I m \omega / \nu_{0}$ versus $k_{z} r_{b}$ obtained numerically from Eq. (15) for several values of $\Gamma_{D}>\Gamma_{D}^{*}=0.364$. 
The Princeton Plasma Physics Laboratory is operated by Princeton University under contract with the U.S. Department of Energy.

\author{
Information Services \\ Princeton Plasma Physics Laboratory \\ P.O. Box 451 \\ Princeton, NJ 08543
}

Phone: 609-243-2750

Fax: 609-243-2751

e-mail: pppl_info@pppl.gov

Internet Address: http://www.pppl.gov 